\title{
Erratum to: Specific disruption of Lnk in murine endothelial progenitor cells promotes dermal wound healing via enhanced vasculogenesis, activation of myofibroblasts, and suppression of inflammatory cell recruitment
}

\author{
Jun Hee Lee ${ }^{1}$, Seung Taek $\mathrm{Ji}^{2}$, Jaeho Kim³ ${ }^{3}$ Satoshi Takaki ${ }^{4}$, Takayuki Asahara ${ }^{5}$, Young-Joon Hong ${ }^{6 *}$ \\ and Sang-Mo Kwon ${ }^{2^{*}}$
}

\section{Erratum}

Unfortunately, after publication of this article [1], it was noticed that Fig. 4 was incorrect. Panels B and D contained incorrect graphs. The corrected Fig. 4 can be seen below and the original article has been updated to correct this.

\begin{abstract}
Author details
'Department of Pharmacology and Toxicology, University of Alabama at Birmingham School of Medicine, Birmingham, AL 35294, USA. ²Department of Physiology, Laboratory for Vascular Medicine and Stem Cell Biology, Medical Research Institute, School of Medicine, Pusan National University, Yangsan 626-870, Republic of Korea. ${ }^{3}$ Research Institute of Convergence Biomedical Science and Technology, Pusan National University School of Medicine, Yangsan, Republic of Korea. ${ }^{4}$ Department of Immune Regulation, Research Centre for Hepatitis and Immunology, Research Institute, National Centre for Global Health and Medicine, Chiba, Japan. ${ }^{5}$ Department of Regenerative Medicine Science, Tokai University School of Medicine, Kanagawa, Japan. ${ }^{6}$ Division of Cardiology of Chonnam National University Hospital, Cardiovascular Convergence Research Center Nominated by Korea Ministry of Health and Welfare, Gwangju 501-757, Republic of Korea.
\end{abstract}

Received: 2 February 2017 Accepted: 3 February 2017

Published online: 09 March 2017

\section{Reference}

1. Lee JH, Ji ST, Kim J, Takaki S, Asahara T, Hong Y-J, Kwon S-M. Specific disruption of Lnk in murine endothelial progenitor cells promotes dermal wound healing via enhanced vasculogenesis, activation of myofibroblasts, and suppression of inflammatory cell recruitment. Stem Cell Research \& Therapy. 2016;7:158. http://doi.org/10.1186/s13287-016-0403-3.

\footnotetext{
* Correspondence: hyj200@hanmail.net; smkwon323@hotmail.com ${ }^{6}$ Division of Cardiology of Chonnam National University Hospital, Cardiovascular Convergence Research Center Nominated by Korea Ministry of Health and Welfare, Gwangju 501-757, Republic of Korea

${ }^{2}$ Department of Physiology, Laboratory for Vascular Medicine and Stem Cell Biology, Medical Research Institute, School of Medicine, Pusan National University, Yangsan 626-870, Republic of Korea
} 
a

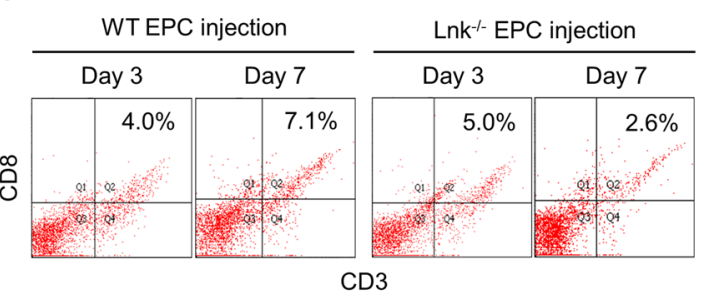

C
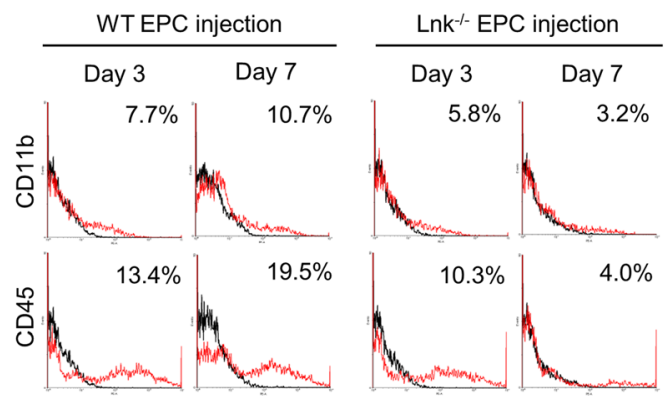

b

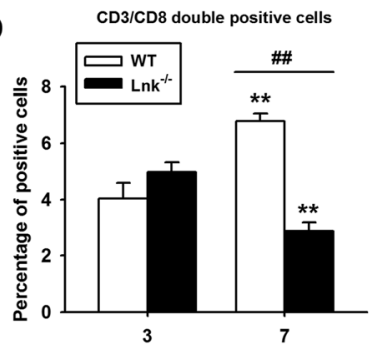

d

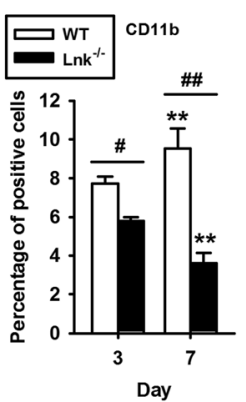

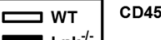
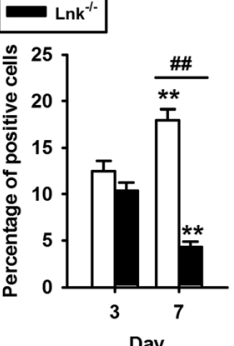

Fig. 4 A transplant of Lnk-deficient EPCs suppresses the recruitment of inflammatory cells. After injection of wild-type (WT) and Lnk-deficient EPCs into wound sites, wound tissues were analyzed to determine the recruitment of cytotoxic T cells (CD3- and CD8-positive cells), macrophages (CD11 b-positive cells), and neutrophils (CD45-positive cells) on postoperative days 3 and 7. a The recruitment of cytotoxic T cells in wound tissues was assessed by FACS analysis. b The percentage of CD3/CD8 double-positive cells on postoperative days 3 and 7 . Values are mean \pm SEM; ${ }^{* *} p<$ 0.01 compared to postoperative day 3, respectively, and \#\#p<0.01 compared to injection with WT EPCS. c The recruitment of macrophages and neutrophils to wound tissues was assessed by FACS analysis. $\mathbf{d}$ The percentage of CD11b-and CD45-positive cells on postoperative days 3 and 7 . Values are mean $\pm \mathrm{SEM}_{*}^{* * *} p<0.01$ compared to postoperative day 3, respectively, $\# p<0.05$ and \#\#p $<0.01$ compared to injection with WT EPCS] 